SUBMUCOUS FIBROID; FIBROID UTERUS; CURETTEMENT AND ANTERIOR FIXATION.*

BY E. E. MONTGOMERY, M.D.

Professor of Gynecology, Jefferson Medical College; Gynecologist to St. Joseph's and Jefferson Hospitals; Ex-President of the Penusylvania State Medical and Pliladelphia Obstetrical Societies.

PHILADEJAHIA, PA.

Submucous fibroid.--One week ago I brought this patient before you, and removed a fibroid growth which had been extruded from the cavity of the uterus into the vagina. This condition is recognized as submucous fibroid, or submucous polypus. The tumor, hung by a pedicle, was very easily twisted off ; but the principal difficulty was in the subsequent removal of the tumor. The patient has done well; there has been no abnormal range of temperature, and her condition is excellent. She sits up to day. Such growths are not unfrequently a source of considerable difficulty in diagnosis. Thus, where the pedicle, or a portion of the tumor is closely constricted by the uterus and sloughing or gangrene of a portion of the tumor results, which causes very profuse discharge, not infrequently hemorrhages, the physician, unless careful in making examination, is led to believe that a malignant disease exists. The digital examination, however, is usually sufficient to disclose the fact that the carious or necrotic tissue is only the lower portion of the tumor, while the upper part will be smooth, which is not characteristic of malignant disease.

Fibroid uterus.-I bring before you another patient with a pelvic growth of a different class. She is 34 years of age and married. Her father is living, and in good health at 82 years of age. Her mother died during confinement, when the patient was 2 years of age, and one sister died at 42 . She had scrofula when young, but the immediate cause of her death was some abdominal trouble. There are two brothers and two sisters living, in good health. The patient has had measles, mumps and scarlet fever dur. ing her childhood, diphtheria at the age of 14, from all which she made a good recovery. Puberty occurred at the age of 12 , the menstrual function being normal until her present trouble. She was married at $2 \check{5}$, but has never been pregnant. Six years ago she had an attack of grippe, which lasted her nine weeks, and during it she had attacks of severe pain in the region of the left ovary, and profuse vaginal discharge. Her family physician asserted that the left ovary was enlarged to the size of a walnut. The patient has had other attacks which have ceased under treatment, since which she has enjoyed excellent health and been free from pain, until two years ago; then she fell backward from a step-ladder and struck her back over the sacrum. Following this injury she suffered from dysmenorrhea, and first noticed an amount of blood at the menstrual periods slightly in excess of that lost in previous years. During the last year she has grown steadily worse, menorrhagia increasing, becoming almost uncontrollable, associated with nausea and vomiting, which required her to remain in bed two or three days out of a month. She has even resorted to opiates to be free from the pain. She is free from pain during her intermenstrual period. The discharge is not offensive, the patient

\footnotetext{
* A clinical lecture delivered at the Jefferson Medical College.
}

is well nourished, and has not lost flesh. She has no trouble with micturition, appetite is good, bowels regular, tongue clean, heart and lungs normal. The urine has an acid reaction, contains neither albumin nor sugar, has a specific gravity of 1021, and shows two grains of urea to each ounce. During the time she has been in the house she has menstruated profusely, pain is quite severe, but not so severe as she has often experienced. She has not required the use of opiates since her entrance.

This patient presents a history of having undergone an injury two years ago, subsequent to which there was an attack of dysmenorrhea, the occurrence of increased menstrual flow, amounting at times to hemorrhage. During this time, there has been an enlargement of the abdomen, and upon examination we find a mass which extends to the umbilicus, situated in the median line, not very regular in outline, presenting a number of nodules. By vaginal or conjoined examination we find the mass extends into the pelvis behind the uterus. That it is continuous with that organ above is evident from the fact that pressure on the mass above causes movement in the one below. It is firm, hard, resistant, and presents not the slightest sign of elasticity or fluctuation. Movement of the mass above with the hand upon the abdomen moves the cervix, which is pushed forward.

You will ask, what is the growth that causes such symptoms? In arriving at a diagnosis it is important to keep before you a careful synopsis of the various conditions which might possibly cause such symptoms, otherwise we may overlook the existence of cer. tain signs which, if recognized, would afford us infor. mation as to the diagnosis and appropriate course of treatment. In summing up, then, the conditions which have produced the symptoms present, we must remember the organs in that part of the body in which this tumor is found, and which necessarily afford the source from which it has originated. It is true that other organs, from increased weight, may gravitate into the pelvis. A very large liver may so stretch its attachments as to extend into the pelvis. A kidney that becomes large or from insufficient attachment becomes known as a floating kidney, may be situated at the bottom of the pelvis. Intestinal tumors, as sarcoma or an omental tumor, may occupy this situation. We have to consider the organs that are in the pelvis, the uterus, ovaries, tubes, rectum, and the possibility of growths from the retrouterine or retroperitoneal tissue. We should not forget the possibility of the patient suffering from an accumulation of fecal matter, as that not unfrequently is a cause of error in diagnosis and has led to operation, when a dose of castor-oil would have been sufficient to have entirely removed it, and rendered the diagnosis certain. In this patient, the tumor is situ. ated in the pelvis and extends upward. If it were the liver we would find a line of dulness continuous from a point above and downward toward the pelvis. On the contrary, the line does not extend above the umbilicus and we are able to indefinitely outline the upper part of this mass. Further, then, we are unable to trace any outline of the fundus of the uterus with the finger in the vagina and the hand over the abdomen. Again, we find the cervix passes directly into the mass. The cervix does not lie to one side of it, but is pushed forward up against the pubic bone and the continuation of the mass with the, cervix demonstrates that the tumor is uterine in its origin. 
In the possibility of uterine tumor we have to consider various forms; thus, fibroid growths, sarcoma or carcinoma of the uterus. The cervix in the great majority of cases is the seat of malignant disease, which forms either as epithelioma, carcinoma, a hard, resist. ing mass, or which may later, through proliferation, break down as an ulceration upon the cervix. Again. malignant disease may begin in the cervical canal or cavity of the uterus, but carcinoma or sarcoma is exceedingly rare. In carcinoma or epithelioma we do not have marked enlargement of the uterus. The body in such cases remains small. In sarcoma the uterus becomes large, but does not attain to the size of the organ of this patient, without more or less breaking down; it does not present the firm resisting character to be felt in this growth, so we are brought to the consideration of the fact that we here have a condition of the uterus known as fibroid degeneration or fibroid growth of the uterus. A week ago I told you we had different varieties of fibroid growth according to the situation. Thus, one found in the cavity of the uterus, covered with mucous membrane, be comes known as a submucous fibroid; when situated equally distant from the internal and the external wall, involving the wall of the organ, it becomes known as mural or interstitial fibroid; others situated near the external layer, as they increase in size are pushed out of the wall beneath the peritoneum, and are known as extramural fibroids, also called subperitoneal. Tumors are also named according to the relation of the uterus It may be extruded from the uterus, hanging by a pedicle, known as a polypus. The term "polypus" is used more particularly with reference to those which are extruded into the cavity of the organ, while those situated in the external surface are known as pedunculated subserous fibroids.

In preparing this patient for operation we have the parts thoroughly cleansed, not only on the external surface through which the incision will be made into the abdomen, but the vulva and vagina as well. The latter is scrubbed thoroughly with tincture of green soap and creolin, irrigated with sterile water, and finally washed with alcohol. One purpose in doing this is that more than likely in the removal of the growth it may be necessary to enter the vagina, and we prefer to have it thoroughly cleansed so as to pre. vent the possibility of infection being derived from it. The operation will consist in the removal of the entire tumor, possibly including the greater, or entire part of the uterus.

Making an incision through the abdominal wall, we are careful to secure the bleeding vessels before we enter the peritoneal cavity. The vessels here are larger, owing to the presence of the pelvis, the abdom. inal vessels becoming more distended. This gives rise to more bleeding than in ordinary conditions. We pick up the peritoneum and cut it between forceps so as to avoid the possibility of injuring a knuckle of intestine situated in front. Such a knuckle of intestine would be very readily injured or possibly omentum incised, requiring additional time to suture it and secure bleeding vessels. Having cut through the peritoneum I introduce my finger to examine the tumor, which is found quite firmly fixed. There have been attacks of inflammation which have bound this tumor down and made it more difficult to accomplish its removal. As a preliminary step I enlarge the wound upward so as to give more room, and a num. ber of sponges are introduced to hold back the intes- tines. In these operations it is sometimes difficult to release the tumor from its bed; the tumor impacted in the pelvis, suction is very marked. This tumor is found difficult to raise. I expedite this a little by splitting through the anterior wall and pushing back the envelope. We in this way practically accomplish partial enucleation of the tumor. It is easier to do this than to separate the firm adhesions to its capsule. I still further enucleate the mass and cut smaller masses out, reducing the size of the mass until weare better able to get around it. Such tumors from pressure in the pelvis not unfrequently become molded to the surface, and you see one which I am now with. drawing, which has been flattened out from its pressure upon the tumor in front of it. We find a cyst of the left tube, which is probably the tube and ovaryyou remember the patient gave a history of having pain on that side.

These are the cases that try men's souls. Everything is matted together and we have to work down carefully, knowing there are important vessels which may be injured. As the tumor is fairly well fixed posteriorly, I cut through the peritoneum in front and push off the bladder, then sutures are introduced latterally to secure the uterine and ovarian arteries, after which we can proceed with our dissection, making sure that we have the mass removed. Very frequently we find the appendix is firmly adherent to it, as in this patient, when I tie it off. This enables us to bring up the fibroid growth which is situated below in the pelvis. We have in this patient to be very particular as to the relation of the parts, for everything is displaced. I am here pulling up a structure which is the round ligament or the ureter, I am uncertain which. Exploring it and the direction which it takes, we find it is the round ligament, considerably larger than usual. If we always had a round ligament the size of this, there would be no difficulty in securing the uterus forward by using it as a support. We now pass the ligature deeply at the side of the uterus to secure the uterine artery. As this ligature is tied, I ask my assistant to take off forceps on this side, which shows that the bleeding vessel has been secured. A ligature is placed on the other side of the stump in a similar manner. It is an important consideration in this case to completely secure hemostasis so that important vessels will not continue to bleed. Knowing where these vesssels come off, we are enabled to pass our ligatures, surround them, and cut off the supply. In some cases, the better plen of procedure instead, would be to ligate en masse or ligate the internal iliac arteries. When the latter is done, it will be found that the bleeding is controlled in the pelvis; pads are now removed and the cavity thoroughly irrigated. There is a tumor situated on the right side, which we enucleate, removing the corresponding tube. A portion of the tumor is filled with blood from the sac that is left. Proceeding further we count the sponges.

Hot water has an excellent effect as a styptic, causes the vessels to contract, and is a good stimulant to the patient. We use for the purpose a normal salt solution, because if we use simply hot water it will cause the epithelial covering of the peritoneum to swell up and render it unfit for the absorption from the peritoneum later. Now we place a hot towel over the intestines to keep them from being exposed, place the patient in the Trendelenburg posture and use an electric light. 'Thus we can thoroughly examine the cavity. Having examined and found that hemor. 
rhage is well controlled we proceed to suture the peritoneum over the stump, shutting it out from the general peritoneal cavity. This is done by a continuous catgut suture, beginning on one side of the pelvis and suturing the peritoneum across the stump to the other side. These sutures are introduced in such a way as to make pressure upon the end of the stump and thus secure additional bemostasis. The procedure is a somewhat tedious part of the work, but very important. We bring these surfaces together, for upon securing the control of the hemorrhage the favorable result very much depends. I again irrigate with normal salt solution, leaving a considerable portion of it remaining in the abdominal cavity. The wound is closed with a figure- 8 stitch, by which the abdominal wall and the aponeurosis are brought in apposition, and the ends brought out through the skin and superficial fascia, so that they have all the advantage of the mattress suture, with the ability to remove it. I am particular in introducing this suture that it picks up the aponeurosis and brings it well over the muscle, so that we will have a good firm ventrum, otherwise the patient will likely suffer from ventral hernia. The sutures are introduced about half an inch apart.

Curettement and anterior fixation.-This patient is 35 years of age. Her mother died of typhoid fever at 31 and her father at 36 . She has lost one brother from scarlet fever, while other members of the family enjoy good health. She had measles and pertussis during infancy. Puberty occurred at the age of 12 The cervix and perineum have been torn and subsequently repaired. Fifteen months after her labor she had a miscarriage at two months. Seven years ago she had a miscarriage at four months, after which preguancy has not recurred. Menstruation is regular, leucorrheal discharge marked at times, but her general health has been good. She has had aching pain in the sacral region and lower part of the abdomen. This pain now precedes and follows menstruation. The establishment of the flow ceases to alleviate the suffering; headache is almost constant. Six months ago she was confined to bed from nervous prostration induced by uterine trouble. The symptoms now are much aggravated, and she suffers from constant distress, which has led to convulsions. These convulsions are likely to occur at the menstrual pe. riud. On examination we find the uterus retroflexed, and there is considerable metritis.

I shall dilate the uterus by bougies. In introducing the first one it must be carefully accomplished to see that it pusses in the proper direction; the instruments as you notice pass backward. Having accomplished the dilatation we curette the cavity, going over the lining membrane in different directions to make sure it is completely scraped, using a flush curette so the fluid will wash away the debris as the operation proceeds. Curettement is followed by packing the cavity with iodoform gauze. It serves as a drain, keeps the surfaces separated, promotes the throwing out of plastic material and sealing up of the abraded surfaces. An incision is made in the lower part of the abdomen, through which to raise up the uterus and draw it forward. A short incision is employed. Old adhesions binding down the uterus posteriorly are broken up, and the fundus seized. A fine silk thread suture is passed through the peritoneum on one side through the fundus of the uterus and brought out through the peritoneum on the opposite side. This is to form a peritoneal band which will permit the mobility of the uterus and yet will restrain it from falling backward. The wound is closed with a figure8 suture.

\section{DERMATOLOGIC DIFFICULTIES*}

BY L. DUNCAN BULKLEY, A.M., M.D.

Attending Physician to the New York Skin and Cancer Hospital; Consult. ing Physician to the New York Hospital, etc.

NEW YORK CITY.

The difficulties of dermatology are thought by many to be very great. Apparently the general practitioner often regards the whole subject as almost hopeless, and is frequently content with a very limited consideration of diseases of the skin. The difficulties in the way of mastering completely all the possibilities of dermatology are indeed not light, and even the most experienced will often find trouble in diagnosis and disap. pointment in treatment. To attempt to point out all dermatologic difficulties would be to produce a textbook on diseases of the skin, so no attempt will be here made to cover all the ground; but it may not be without advantage to direct attention to a few of the points where special difficulties occur, and to make a few practical suggestions in regard to overcoming them. We may briefly consider them under the heads of diagnosis, prognosis and treatment.

Diagnosis. - While in a certain proportion of cases it is relatively easy to make a correct diagnosis, to one who has any knowledge of this class of diseases, it must be acknowledged that in a considerable propor. tion of cases there must always be some doubt, until analytic reasoning has established the exact nature of the case. As correctness in diagnosis is the first essential of successful treatment, in diseases of the skin, almost more than in other branches of medicine, a little thought may be given to this dermatologio difficulty.

1. The examination should be made in the most complete and thorough manner possible: no hasty or superficial glance will enable even the skilled observer to arrive at a positive diagnosis. The practitioner should never be satisfied with inspecting only a portion of the eruption present, but he must insist upon examining the whole of the diseased surface; and it is also important to examine the neighboring healthy skin as well.

2. To observe diseases of the skin satisfactorily it is necessary that there should be good light; for this purpose daylight is almost essential, and plenty of it, preferably from a northern exposure. It is almost impossible to judge correctly of the color of skin lesions by artificial light. A good lens, about two inches in diameter, is often of great assistance in bringing out clearly the separate features of an eruption. The microscope should always be used in doubtful cases of the vegetable parasitic eruptions.

3. Care and patience must be exercised, in very many cases, in analyzing the elements of the eruption and determining exactly the disease to which they belong and the indications present for treatment. In making the diagnosis of an eruption there are two methods by which the end can be arrived at; these should be employed together, in order to obtain any absolute accuracy. The first is by recognizing the actual characters or features of the disease present,

* Presented to the Section on Cutaneous Medicine and Surgery, at the Forty-ninth Annual Meeting of the American Medical Association, Licid at Denver. Colo., June 7-10, 1898 